\title{
Master Symmetries and Associated Conservation Laws of Certain Nonlinear Evolution Equations
}

\author{
K. Thilaga and P. S. Bindu \\ Department of Physics, Lady Doak College, Madurai 625002, Tamil Nadu, India
}

\begin{abstract}
In this paper, we have systematically investigated certain nonlinear evolution equations such as Burgers Fisher equation, Inviscid Burgers equation, Satsuma equation and Fokas Yortsos-Rosen equation by determining the master symmetries, time dependent symmetries and the associated conservation laws. As the Burgers Fisher equation and Satsuma equation are diffusive, they do not possess infinitely many conservation laws and are reduced to the linear heat equation. Besides, certain systems which do not admit master symmetries, are also identified.
\end{abstract}

Keywords: Conservation laws, Master symmetries, Nonlinear evolution equations.

\section{Introduction}

Symmetries and conservation laws play a very important role in deciding the integrability of nonlinear partial differential equations (PDEs) [1]. During the last two decades, Lie's one parameter continuous point transformation has been effectively used to find a class of particular solutions to PDEs. Further this method has also been used to reduce nonlinear PDEs to ODEs of Painlevé type, provided if the system is an integrable one.

With the new discovery of so many completely integrable evolution equations, there is a growing demand for simple, transparent and direct methods to obtain these quantities in an explicit form [2]. Thus, Fuchssteiner introduced a new class of symmetries called master symmetries in which space variables are involved explicitly. Master symmetries are an interesting class of symmetries admitted by nonlinear PDEs possessing solitons, which involves both dependent and independent variables and are related with generalized symmetries [2,3,4]. A master symmetry (of degree $\mathrm{n}$ ) for a nonlinear PDE, is a derivation in the Lie algebra of vector fields having the property that $\mathrm{n}$ fold applications leaves the commutator of the flow under consideration invariant [4]. The existence of master symmetries is one of the characteristics of completely integrable nonlinear pdes $[2,3]$.

When a nonlinear PDE admits a master symmetry, it usually admits infinitely many other symmetries where the successive elements involve independent variables, dependent variables as well as spatial derivatives of the dependent variables. That is, the existence of master symmetry implies the existence of time independent generalized symmetry, which in turn leads to infinitely many such symmetries. This is true for a large class of evolution equations in one space dimension. And this is one of the advantages of constructing master symmetries, since they guarantee the existence of generalized symmetry [3]. Moreover the importance of time dependent symmetries is that they can be useful for constructing new conservation laws from a given one.

The identification of integrable systems and the study of the relation between integrability and the symmetry structure of the system have been considered in the past years, by several authors [5, 6]. Investigations have also revealed the explicit construction of the basic invariants such as conserved quantities, symmetries, master symmetries and recursion operators for the nonlinear lattice systems [7]. Many Studies have been carried out to determine the travelling wave solutions for the Burgers, Fisher, Huxley equations and combined forms of these equations. [8,9]

In this paper, we present the construction of master symmetries, generalized symmetries, time dependent symmetries and conservation laws of systems such as Burgers Fisher equation, Satsuma equation, Fokas Yortsos-Rosen equation, Inviscid Burgers equation. Later we also point out the systems that do not admit master symmetries.

\section{Master Symmetries And Conservation Laws For Certain Evolution Equations}

In this section, we investigate the master symmetries, time dependent and independent symmetries and the associated conservation laws for evolution systems like the Burgers-Fisher equation, Inviscid Burgers equation, Fokas-Yortsos Rosen equation and the Satsuma Equation. 


\subsection{Burgers-Fisher equation}

Fisher first proposed the well-known equation, encountered in various disciplines, as a model for the propagation of a mutant gene with $q(x, t)$ displaying the density of advantage. The most general form of the Fisher equation is called the Burgers- Fisher equation. Burgers-Fisher type equations describe the interaction between reaction mechanism, convection effect and diffusion process. This equation has a wide range of applications in plasma physics, fluid physics, capillary-gravity waves, nonlinear optics, chemical physics and population dynamics.

The Burger's Fisher equation is of the form [10]

$$
q_{t}=K_{1}, \quad K_{1}=\alpha q q_{x}+q_{x x}+\beta q-\beta q^{2} .
$$

where $\beta$ is a constant. Here $K_{1}$ remains invariant upto a multiplicative constant under the transformation $q \mapsto \alpha q, x \mapsto \alpha x$ and admits the scaling

$$
\tau_{0}=x q_{x} .
$$

The class of solutions of the equation $\tau_{0}=\left\lfloor q_{x_{j}}, f\right\rfloor_{L}$ depends on the form of $f$, where $[,]_{L}$ represents the Lie commutator. If $f(q) \in \mathfrak{R}\left\{q, \partial^{-1} q, x\right\}$ then we find

$$
f=\frac{x^{2}}{2} q_{x}+g(q)
$$

where $g$ is an arbitrary function in $\mathfrak{R}\left\{q, \partial^{-1} q\right\}$. We define the master symmetry as $\tau=\left[K_{1}, f\right]_{L}+\tau_{1}$. Let $\tau_{1}, g=0$. Then we get

$$
\tau=K_{1}^{\prime} f-f^{\prime} K_{1}=\alpha x q q_{x}+q_{x}+2 x q_{x x}
$$

from which we determine the function $K_{2}$ using $\left[K_{1}, \tau\right]_{L}$ :

$$
\begin{aligned}
K_{2} & =K_{1}^{\prime} \tau-\tau^{\prime} K_{1}=\alpha^{2} q^{2} q_{x}-2 \alpha x q_{x} q_{x x}+4 \alpha q q_{x x} \\
& +2 \alpha q_{x}^{2}+4 q_{x x x}+\alpha \beta x q^{2} q_{x}-\alpha \beta x q q_{x}+4 \beta x q q_{x x} .
\end{aligned}
$$

We find that $K_{2}$ commutes with $K_{1}$ iff $\alpha$ and $\beta=0$, i.e.,

$$
\left[K_{1}, K_{2}\right]=0 \text { when } \alpha, \beta=0 .
$$

and hence $K_{2}$ is a symmetry of the given PDE.

Using (6), the Burgers Fisher equation reduces to the linear heat equation

which admits the master symmetry

$$
q_{t}=q_{x x}
$$

$$
\tau=q_{x}+2 x q_{x x} .
$$

Further we find the next time independent symmetry $K_{3}$ as

$$
K_{3}=\left[K_{2}, \tau\right]_{L}=K_{2}^{\prime} \tau-\tau^{\prime} K_{2}=24 q_{x x x x}
$$

Here, the master symmetry $\tau$ generates the odd time-independent generalized symmetries $K_{2 j+1}$ of the BurgersFisher hierarchy $K_{2 j+1}=\left\lfloor K_{j}, \tau\right\rfloor_{L}$, thereby leading to infinitely many symmetries.

Further, the Burgers-Fisher equation (1) can be written in a form of the conservation law

$$
D_{t}(q)+D_{x}\left(-q_{x}\right)=0
$$

where,

$$
T_{0}(q)=q \text { and } T_{1}(q)=-q_{x}
$$

are the conserved density and flux respectively. And the corresponding constant of motion is $\int q d x$. This describes the law of conservation of mass if $q$ is interpreted as density and as conservation of thermal energy if $q$ is interpreted as the temperature. Since the Burgers-Fisher equation models the propagation of the gene with $q(x, t)$ displaying the density of propagation, (10) represents the conservation of mass. And Since the BurgersFisher equation is diffusive, it does not possess infinitely many conservation laws. Furthermore, $\left\{T_{0}^{\prime} K_{j}, T_{1}^{\prime} K_{j}\right\} \sim 0$ and hence none of the symmetries $K_{j}$ yielded new conservation laws. 
Also, we have found that the Burgers Fisher equation possesses $x, t$-dependent symmetry $\sigma_{j} \in \mathfrak{R}\left\{q, \partial^{-1} q, t, x\right\}$ given by

$$
\sigma=t K_{2}+\tau=4 t q_{x x x}+q_{x}+2 x q_{x x}
$$

\subsection{Inviscid Burgers equation}

Burgers equation is one of the basic partial differential equations of fluid mechanics [11]. It occurs in various fields of applied mathematics, such as modeling of gas dynamics and traffic flow. Originally Burgers equation was introduced and used as a simplistic model for one dimensional turbulence. The original equation, $q_{t}+q_{x x}+v q_{x x}$ which shall be referred to as viscous Burgers equation, includes a dissipative viscous term. By removing the viscous term, Inviscid Burgers equation is obtained. It is well established that Inviscid Burgers equation forms discontinuities (shock waves) in finite time, determined by initial conditions and is given by

$$
q_{t}=K_{1}, \quad K_{1}=-q q_{x}
$$

which remains invariant upto a multiplicative constant under the transformation $q \mapsto q, \quad x \mapsto \alpha x$ admits the scaling $\tau_{0}=x q_{x}$, and it may be verified that the function $f=\frac{x^{2}}{2} q_{x}$ satisfies the equation $\tau_{0}=\left\lfloor q_{x_{j}}, f\right\rfloor_{L}$. Then we obtained the master symmetry $\tau$ as

$$
\tau=\left[K_{1}, f\right]_{L}=K_{1}^{\prime} f-f^{\prime} K_{1}=-x q q_{x} .
$$

From (13) and (14) the symmetry $K_{2}$ is determined as

$$
K_{2}=\left[K_{1}, \tau\right]_{L}=K_{1}^{\prime} \tau-\tau^{\prime} K_{1}=q^{2} q_{x} .
$$

The equation (13) commutes with $K_{1}$ i.e.,

$$
\left[K_{1}, K_{2}\right]=0 \text {. }
$$

Thus the Inviscid Burgers equation (13) admits the master symmetry

$$
\tau=-x q q_{x} .
$$

We would like to remark that, the master symmetry $\tau$ generates the odd time-independent generalized symmetries of the Inviscid Burgers hierarchy

$$
K_{3}=\left[K_{2}, \tau\right]_{L}=K_{2}^{\prime} \tau-\tau^{\prime} K_{2}=-q^{3} q_{x} .
$$

The Inviscid Burgers equation admits infinitely many symmetries $K_{j}$ and local conservation laws. The Inviscid Burgers equation is in a form of the conservation law

$$
D_{t}(2 q)+D_{x}\left(q^{2}\right)=0
$$

with the constant of motion [12]

$$
\frac{d}{d t} \int_{a}^{b} T_{0} d x=0
$$

The above equation represents the rate of change of the integrated density over an interval that depends only on the flux through its end points. Using (19), the integrated conservation law (20) takes the form

$$
\frac{d}{d t} \int_{a}^{b} 2 q(t, x) d x=\left[q(t, a)^{2}-q(t, b)^{2}\right]
$$

which represents the model for compressible fluid flow in a pipe, where $\int 2 q d x$ represents the total mass of the fluid. Thus the conservation law indicates that the basic mass is conserved. Since $\left\{T_{0}^{\prime} K_{j}, T_{1}^{\prime} K_{j}\right\} \sim 0$, none of the symmetries $K_{j}$ yield new (nontrivial) conservation laws.

Furthermore, the Inviscid Burgers equation possesses time dependent symmetry given by

$$
\sigma=t K_{2}+\tau=t q^{2} q_{x}-x q q_{x} .
$$

The symmetry $\sigma$ gives rise to the sequence of conservation laws associated with the Inviscid Burgers equation:

$$
\begin{aligned}
& \left\{T_{0}^{(1)}, T_{1}^{(1)}\right\}=\left\{3 q^{2}, 2 q^{3}\right\}, \\
& \left\{T_{0}^{(2)}, T_{1}^{(2)}\right\}=\left\{4 q^{3}, 3 q^{4}\right\},
\end{aligned}
$$




$$
\begin{aligned}
& \left\{T_{0}^{(3)}, T_{1}^{(3)}\right\}=\left\{5 q^{4}, 4 q^{5}\right\}, \\
& \left\{T_{0}^{(4)}, T_{1}^{(4)}\right\}=\left\{6 q^{5}, 5 q^{6}\right\} .
\end{aligned}
$$

\subsection{Fokas-Yortsos Rosen equation}

In general the nonlinear reaction--diffusion systems give rise to very many complex patterns and their analysis becomes extremely complicated. Combined numerical and analytical approaches are needed to elucidate the underlying structures. Fokas-Yortsos-Rosen equation is one of such physically interesting nonlinear diffusion systems. The Fokas-Yortsos Rosen equation is used as a model that describes the fluid flow in a homogeneous permeable solid [13]

$$
q_{t}=K_{1}, \quad K_{1}=d q^{2} q_{x x}-q^{2} q_{x}
$$

The above function remains invariant upto a multiplicative constant under the transformation $q \mapsto q, \quad x \mapsto \alpha x$

and it admits the scaling $\tau_{0}=x q_{x}$. The function $f$ obtained as $\frac{x^{2}}{2} q_{x}$ satisfies the equation $\tau_{0}=\left\lfloor q_{x_{j}}, f\right\rfloor_{L}$.

We determined the master symmetry $\tau$ as

$$
\tau=\left[K_{1}, f\right]_{L}=d q^{2} q_{x}+2 x d q^{2} q_{x x}-x q^{2} q_{x} .
$$

The function $K_{2}$ is obtained as

$$
K_{2}=12 d^{2} q^{3} q_{x} q_{x x}+4 d x q^{3} q_{x} q_{x x}+2 d^{2} q^{2} q_{x}^{3}+4 d^{2} q^{4} q_{x x x}-4 d q^{3} q_{x}^{2}-4 d q^{4} q_{x x}-2 d x q^{2} q_{x}^{3}+q^{4} q_{x}
$$

The equation (26) commutes with $K_{1}$ when $d=0$. Thus the Fokas-Yortsos Rosen equation admits the master symmetry $\tau=-x q^{2} q_{x}$.

As already pointed out, the master symmetry $\tau$ generates the odd time-independent generalized symmetries of the Fokas-Yortsos Rosen hierarchy

$$
K_{3}=\left[K_{2}, \tau\right]_{L}=K_{2}^{\prime} \tau-\tau^{\prime} K_{2}=-q^{6} q_{x} .
$$

The Fokas-Yortsos Rosen equation is in a form of conservation law,

$$
D_{t}(3 q)+D_{x}\left(q^{3}\right)=0
$$

and the corresponding constant of motion is [12]

$$
\frac{d}{d t} \int_{a}^{b} T_{0} d x=0 .
$$

The above equation indicates that there is no net flux into and out of the interval, thus the integrated density is conserved, meaning that it remains constant over a time. Using (28), the integrated conservation law (29) takes the form

$$
\frac{d}{d t} \int_{a}^{b} 3 q(t, x) d x=\frac{3}{2}\left[q(t, a)^{2}-q(t, b)^{2}\right]
$$

Viewing the above equation as a model for fluid flow in a homogeneous permeable solid, the integral on the left hand side represents the total mass of the fluid contained in the interval $[a, b]$. The right hand side represents the mass flow into the interval through its two end points, thus (30) is a mathematical formalization of mass conservation and hence mass is neither created nor destroyed. Since $\left\{T_{0}^{\prime} K_{j}, T_{1}^{\prime} K_{j}\right\} \sim 0$, none of the symmetries $K_{j}$ yield new (nontrivial) conservation laws.

Also the Fokas-Yortsos Rosen equation possesses time dependent symmetry given by

$$
\sigma=t K_{2}+\tau=t q^{4} q_{x}-x q^{2} q_{x} .
$$

The symmetry $\sigma$ gives rise to sequence of conservation laws associated with the Fokas-Yortsos Rosen equation

$$
\begin{aligned}
& \left\{T_{0}^{(1)}, T_{1}^{(1)}\right\}=\left\{2 q^{2}, q^{4}\right\} \\
& \left\{T_{0}^{(2)}, T_{1}^{(2)}\right\}=\left\{5 q^{3}, 3 q^{5}\right\} \\
& \left\{T_{0}^{(3)}, T_{1}^{(3)}\right\}=\left\{6 q^{4}, 4 q^{6}\right\}, \\
& \left\{T_{0}^{(4)}, T_{1}^{(4)}\right\}=\left\{7 q^{5}, 5 q^{7}\right\} .
\end{aligned}
$$




\subsection{Satsuma equation}

The Satsuma equation or generalized Burgers-Huxley equation [14] of the form

$$
q_{t}-q_{x x}+\alpha q q_{x}=\beta q(1-q)(q-v)
$$

where $\alpha, \beta, v$ are the parameters, describes the interaction between convection, diffusion and reaction. The above equation is rewritten as

$$
q_{t}=K_{1}, \quad K_{1}=q_{x x}+\alpha q q_{x}+\beta q(1-q)(q-v)
$$

The function $K_{1}$ remains invariant upto a multiplicative constant under the transformation $q \mapsto q, x \mapsto \alpha x$ admits the scaling $\tau_{0}=x q_{x}$ and its solution is given by $f=\frac{x^{2}}{2} q_{x}$. We determined the master symmetry $\tau$ as

$$
\tau=\left[K_{1}, f\right]_{L}=q_{x}+2 x q_{x x}+\alpha x q q_{x} .
$$

The function $K_{2}$ is defined as

$$
\begin{aligned}
K_{2}= & 4 q_{x x x}+2 \alpha q_{x}^{2}+4 \alpha q_{x x}-2 \alpha x q_{x} q_{x x}+\alpha^{2} q^{2} q_{x}-4 \beta x q_{x}^{2}+12 \beta x q q_{x}^{2} \\
& -4 \beta v x q_{x}^{2}-\alpha \beta x q^{2} q_{x}+\alpha \beta v x q q_{x}+\alpha \beta x q^{3} q_{x}-\alpha \beta v x q^{2} q_{x}
\end{aligned}
$$

The function $K_{2}$ commutes with $K_{1}$ if $\alpha, \beta, v=0$. Thus the Satsuma equation reduces to the linear heat equation, which admits the master symmetry $\tau=q_{x}+2 x q_{x x}$ as that of the Burgers Fisher equation and the results follow thereafter.

In addition to the above, we found that the classical Fisher equation, density dependent reaction and diffusion equation and Kolmogorov-Petrovskii-Piskunov equation does not possesses master symmetry because there exist an uncertain relation between the symmetries $K_{1}$ and $K_{2}$. Thus the integrability of these equations can be determined by using other methods.

\section{Conclusion}

In this paper we have investigated certain evolution equations by determining their master symmetries and associated conservation laws. Since the master symmetry implies the existence of generalized symmetry which in turn leads to infinitely many symmetries, we have determined the time-dependent and the generalized time-independent symmetries and hence the conserved quantities of the systems under consideration.

\section{References}

[1] R. Sarathy and K. M. Tamizhmani, Master Symmetries of Certain Partial Differential Equations, Research Report in Physics, 1990, 20-26.

[2] Benno Fuchssteiner, An Approach To Master Symmetries of Lattice equations, arXiv:solv-int/9807010v2, 1988.

[3] F. Finkel and A.S. Fokas, On the Constructions of Evolution Equations Admitting Master Symmetry, Physics Letters A., 293, 2002, 36-44.

[4] R.Sahadevan and L.Nalinidevi, Generalized, Master and Nonlocal Symmetries if certain deformed Nonlinear Partial Differential Equations, Journal of Nonlinear Mathematical Physics, 17 (4), 2010, 517-538.

[5] George Bluman, Connections Between Symmetries and Conservation Laws, arXiv:math-ph/0511035, 2005.

[6] V. E. Alder, A. B. Shabat and R. I. Yamilov, Symmetry Approach to the integrability problem, Theoretical and Mathematical Physics, 125(3), 2000, 1603-1661.

[7] Zhang Hongwei, Tu Gui Zhang, Walter Oevel and Benno Fuchssteiner, Symmetries, Conserved quantities and Hierarchies for Some Lattice Systems with Soliton Structure, J. Math.Phy, 32, 1991, 1908-1918.

[8] Abdul-Majid Wazwaz, Analytic study on Burgers, Fisher, Huxley equations and combined forms of these equations, Applied Mathematics and Computation, 195 (2), 2008, 754-761.

[9] X Y Wang, Z S Zhu and Y K Lu, Solitary wave solutions of the generalized Burgers-Huxley equation, Journal of Physics A: Mathematical and General, 23 (3).

[10] Guaoliang Cai, Yan Wang and Fengyun Zhang, Nonclassical Symmetries and Group Invariant Solutions of BurgersFisher Equations, World Journal of Modeling and Simulations, 3, 2007, 305-307.

[11] Greg Norgard and Kamran Mohseni, A Regularization of Burgers Equation using a Filtered Convective, arXiv:0806.0400v1 [physics-flu-dyn].,2008

[12] Inna Shingareva carlos Lizarraga-celaya, Solving Nonlinear Partial Differential Equations with Maple and Mathematica, Springer-Wien, New York, 2003

[13] M. Lakshmanan and S. Rajasekar, Nonlinear Dynamics: Integrability, Chaos and patterns, Springer-Verlag, New York, 2003.

[14] Lokenath and Debnath, Nonlinear Partial Differential Equations for Scientists and Engineers, University of Texas Pan-American,USA,2004 